\title{
Fast Analysis of Stop-Band FSS Integrated with Phased Array Antennas
}

\author{
Francesco CAMINITA, Alessio CUCINI, Stefano MACI \\ University of Siena, Department of Information Engineering, Siena, 53100, Italy \\ caminita@dii.unisi.it, macis@dii.unisi.it
}

\begin{abstract}
This paper presents a method for the efficient analysis of multilayer frequency selective surfaces (FSSs) integrated with phased array of open-ended waveguides. The method is based on the assumption that all the periodic surfaces are arranged on the same spatial lattice (of arbitrary shape). The whole structure is represented as an equivalent multi-mode transmission line network, where each interface is characterized by an equivalent Generalized Scattering Matrix (GSM), computed through a fullwave analysis. To reduce the computational effort of the analysis a fast adaptive interpolation algorithm for the scattering matrix entries is included.
\end{abstract}

\section{Keywords}

Frequency selective surface, generalized scattering matrix, open-ended waveguide arrays.

\section{Introduction}

Recently, a growing interest has been devoted to the application of frequency selective surfaces (FSS) [1] for obtaining specific electromagnetic properties, such as artificial surfaces, artificial magnetic conductors, enhanced directivity, electromagnetic band-gap properties. Another field of interest is the integration of FSS with phased array antennas [2], with the purpose of improving the antenna performance (widening of scan region, scan-blindness removal [3], wide angle impedance matching [4]).

In this work, a method is proposed for the analysis of multilayered FSS integrated with phased array constituted by rectangular waveguides. The structure includes a multilayered cover with an arbitrary number of dielectric layers and FSSs. The primary goal is the achievement of an outof-band frequency third harmonic rejection for EMI reduction in complex environments. Additional benefits that can be reached with these structures and will be investigated are wide angle impedance matching and surface wave suppression (for widening of the array blindness-free scan region). The analysis is based on the assumption that the structures are periodic and large with respect to the wavelength. The array excitation amplitude may be uniform or weakly spatially. With these hypotheses, the entire struc- ture can be considered as infinite and periodic; this allows to expand the electromagnetic quantities (fields and currents) in terms of Floquet wave (FW) series, thus, reducing the analysis to a single periodic cell.

The problem is solved by a full-wave analysis of each individual periodic interface (array or FSS) with the pertinent Green's function. From the method of moment matrix, an equivalent multimode Generalized Scattering Matrix (GSM) of the periodic surface is derived [7], which permits to achieve a multimode equivalent network of the entire structure. The blocks interconnection is realized using conventional transmission-line methods. The order of the multimode equivalent network is determined by the number of accessible modes [5], that are those FWs which are responsible of the interaction between two adjacent periodic interfaces. This approach drastically reduces the numerical effort and provides a physical insight into the mechanism of the interaction between the aforementioned structures.

\section{Formulation}

The structure under study is shown in Fig. 1a. The phased array antenna is composed by open-ended rectangular waveguides, arranged on a periodic grid (rectangular or skewed). A multilayered cover is placed in front of the array plane, composed by an arbitrary number of dielectric layers. The layers are numbered from 1 to $N$. The $i$-th dielectric layer has thickness $l_{i}$, relative (complex) permittivity $\varepsilon_{r}$, and relative (complex) permeability $\mu_{r i}$. An FSS may be present between two adjacent dielectric layers. The FSSs are assumed to be perfectly conducting and with zero thickness.

Under the hypothesis of periodicity of the geometry and of the excitation (hypothesis that may be relaxed for slowly varying amplitude excitations) the unit periodic cell approach is applied (Fig. 1b). Thus, the analysis is reduced to that of a transition between a metallic waveguide and a periodic boundary waveguide, with inclusion of dielectric layers and perfectly conducting capacitive/inductive insets. The most conventional approach for this kind of problem consists in the use of the periodic MoM to the entire cell (comprising the waveguide element and the FSSs). Otherwise this approach suffers some drawbacks. First, the com- 
putational effort rapidly increases for structures with multiple FSSs and dielectric layers. Next, if a modification is introduced in the shape of a FSS element, the entire cell needs to be analyzed again. This constitutes a strong limitation, especially during iterative design processes, which usually involve a high number of full-wave steps of analysis.

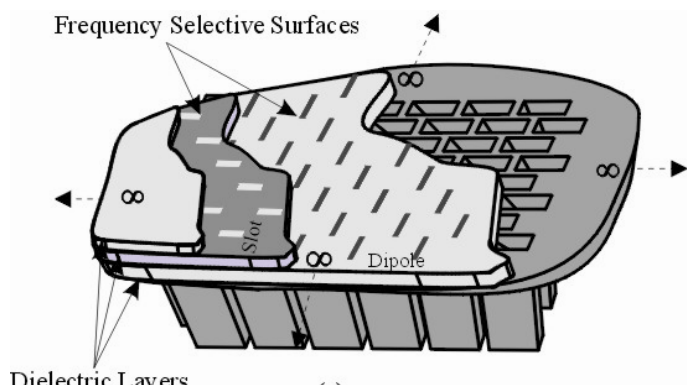

Dielectric Layers

(a)

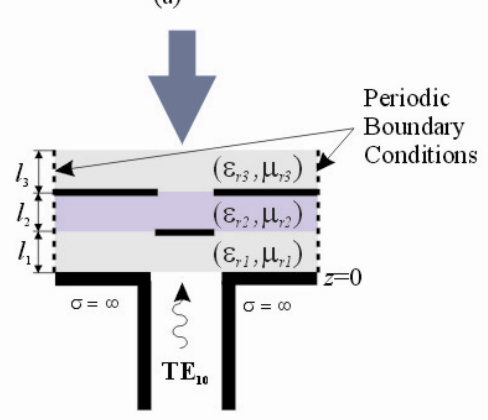

(b)

Fig. 1. Array of open ended waveguides, integrated with multilayer FSS: (a) three-dimensional view and (b) periodic cell side view.

On the basis of the previous considerations, we follow here a different scheme. The MoM analysis is applied separately to each periodic discontinuity of the unit cell (array of open-ended waveguides or FSS), with the goal to describe each transition by the Generalized Scattering Matrix (GSM) [7] as shown in the example of Fig. 2. Once the periodic surfaces are characterized by their GSM, the complete equivalent network, associated to the entire stratification, is solved by the connecting the scattering matrices with equivalent transmission-lines associated to the accessible modes. This technique is similar to the multi-mode equivalent network proposed in [2], but with the use of the equivalent GSM more than admittance matrices for the periodic interfaces.

The size of the matrices depends on the number of accessible modes [5]. This concept is briefly summarized herein after for convenience. Consider a two-layer FSS, as in Fig. 3, composed by an array of patches cascaded with an array of apertures on a metal screen. If the two transition are separated by a dielectric layer whose thickness is $h$ and whose relative dielectric constant is $\varepsilon_{\mathrm{r}}$, the FW modes excited at the patch-type FSS can reach or not the aperture-type FSS depending on the $z$ propagation constant, $k_{z, p q}=\sqrt{\varepsilon_{r} k^{2}-k_{x, p q}^{2}-k_{y, p q}^{2}}$ which influences the exponential $\exp \left(-j k_{z, p q} h\right)$.

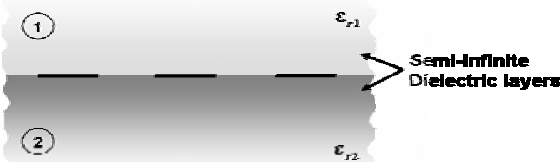

I

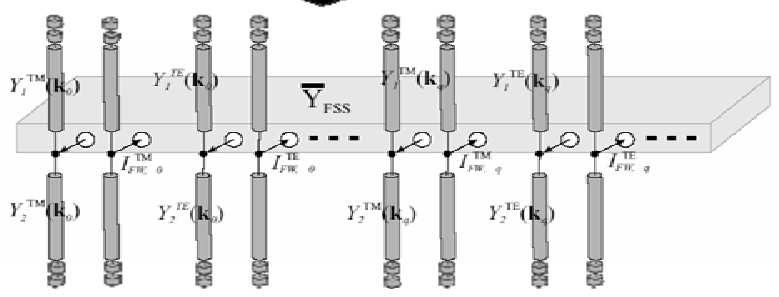

Fig. 2. Equivalent transmission line for a patch-type FSS between two dielectric layers.

The accessible modes may be both propagating or evanescent, and are responsible for the interaction junction between the field. The localized modes are higher-order modes, below cut-off and with high attenuation constant, that do not interact with the aperture-type FSS and take into account the reactive energy near the patch-type FSS. Only the accessible modes need to be included in the multimode equivalent network in order to describe interaction between the two FSS. This approach is generalized to take into account an arbitrary numbers of FSS and the array.

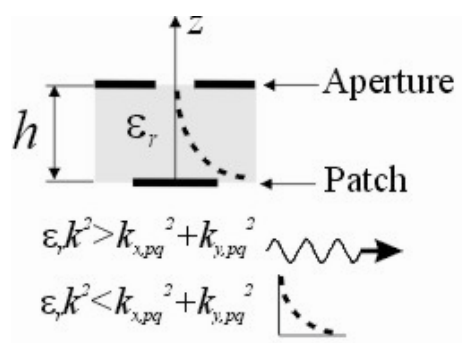

Fig. 3. Pictorial representation of propagating and evanescent modes between two coupled FSSs.

\section{Derivation of the Generalized Scat- tering Matrix}

The derivation of the GSM is provided in [7] and it can be constructed directly from the formulation described in [6] for the analysis of artificial and EBG surfaces based on printed aperture-type and patch-type FSS on a grounded dielectric slab. The approach is based on the MoM formulation for the integral equation of the problem, and on the successive derivation, from the MoM matrix, of the GSM associated to the periodic discontinuity.

Consider a patch-type FSS (Fig.1). By applying the equivalence theorem, an electric current distribution is assumed on the region of the metallic patches, radiating with the Green's function (GF) of the two semi-infinite dielectric regions. By imposing the boundary conditions on the surface of the metallic patches, the Electric Field Integral Equation (EFIE) is derived, as follows: 


$$
\mathbf{E}_{s}(\mathbf{J})+\mathbf{E}_{i m p}=0
$$

where $\mathbf{E}_{\mathrm{S}}$ is the field radiated by the currents $\mathbf{J}$ induced on the dipoles, and $\mathbf{E}_{i m p}=\mathbf{E}_{i n c}+\mathbf{E}_{\text {ref }}$ is the impressed field at the interface (in the absence of printed dipoles), which is given by the sum of the incident $\mathbf{E}_{i n c}$ and reflected $\mathbf{E}_{\text {ref }}$ fields. From here on, the bold characters indicate vectors and the carets indicate unit vectors. By using a Galerkin spectral MoM approach, (1) is reduced to the matrix equation

$$
\overline{\bar{Z}}_{\text {мом }} \bar{I}=\bar{V}
$$

where $\bar{I}$ is the unknown column vector of the expansion coefficients of the currents, $V^{-}$is the known column vector of the complex amplitude of the impressed electric field projected on the MoM basis, and $\overline{\bar{Z}}_{\text {Moм }}$ is the MoM impedance matrix, which can be expressed in the compact form as

$$
\overline{\bar{Z}}_{M o M}=\overline{\bar{Q}}^{H} \overline{\bar{Z}}_{G F} \overline{\bar{Q}}
$$

where $\overline{\bar{Z}}_{G F}$ is a diagonal $2 M \times 2 M$ matrix of the Green's function sampled at the FW wavenumbers (being $M$ the number of FW included in the expansion), $\overline{\bar{Q}}$ is a $2 M \times N$ matrix and $\overline{\bar{Q}}^{H}$ (the superscript $H$ denotes complex conjugate transpose matrix) is an $N \times 2 M$ matrix, whose entries are given by the TM/TE components of the Fourier transform of the basis functions, sampled at the FW wavenumbers.

The MoM vector $\bar{I}$ is written in terms of the $\mathrm{FW}$ modal voltage vector $\bar{I}_{F W}$, i.e., $\bar{I}_{F W}=\overline{\overline{Q_{A}}} \bar{I}$ where $\overline{\overline{Q_{A}}}$ is the $2 M_{A} \times N$ matrix consisting of the first $2 M_{A}$ TM/TE rows of $\overline{\bar{Q}}$. The forcing term vector associated to the incident electric field is also projected onto the FW modal vector via TM/TE decomposition, which leads to $\bar{V}={\overline{\overline{Q_{A}}}}^{H} \bar{V}_{F W}^{i m p}$, where $\bar{V}_{F W}^{i m p}$ is the incident field, and ${\overline{\overline{Q_{A}}}}^{H}$ is the $N \times 2 M_{A}$ matrix obtained by the first $2 M_{A}$ columns of $\overline{\bar{Q}}^{H}$. With $M_{A}$ we denoted the number of accessible FW modes. By using the above projections in the MoM system, we obtain

$$
\begin{aligned}
& \bar{I}_{F S}^{F S S}=\overline{\bar{Y}}_{F W} \bar{V}_{F W}^{i m p}, \\
& \overline{\bar{Y}}_{F W}={\overline{\overline{Q_{A}}}}_{\bar{Z}}^{-1} \overline{\bar{Q}}^{H} .
\end{aligned}
$$

Eq (5) establishes the relationship between impressed modal voltages at the FSS elevation and total modal currents associated to $2 M_{A}$ accessible FW modes. After some algebraic manipulations, following the procedure in [7], the Generalized Scattering Matrix for patch-type FSS between two semi-infinite dielectric regions can be expressed as

$$
\overline{\bar{S}}_{F S S}=\left[\begin{array}{ll}
\overline{\bar{S}}_{11} & \overline{\bar{S}}_{12} \\
\overline{\bar{S}}_{21} & \overline{\bar{S}}_{22}
\end{array}\right]
$$

with the matrix entries defined as follows

$$
\begin{aligned}
& \overline{\bar{S}}_{11}=\overline{\bar{\Gamma}}-\overline{\bar{Y}}_{G F} \overline{\bar{Y}}_{F W}(\overline{\bar{I}}+\overline{\bar{\Gamma}}) ; \\
& \overline{\bar{S}}_{21}=\overline{\bar{S}}_{11}+\overline{\bar{I}} ; \\
& \overline{\bar{S}}_{12}=\overline{\bar{S}}_{22}+\overline{\bar{I}} \\
& \overline{\bar{S}}_{22}=-\overline{\bar{\Gamma}}-\overline{\bar{Y}}_{G F} \overline{\bar{Y}}_{F W}(\overline{\bar{I}}-\overline{\bar{\Gamma}}) ;
\end{aligned}
$$

where $\overline{\bar{I}}$ is identity matrix, $\overline{\bar{Y}}_{G F}=\overline{\bar{Z}}_{G F}^{-1}, \overline{\bar{\Gamma}}$ is the scattering matrix at the dielectric interface.

For an aperture-type FSS a similar approach is applied starting from the Magnetic Field Integral Equation (MFIE). In this case, by applying the equivalence theorem, the FSS is substituted by a continuous, infinitely thin PEC screen with magnetic current distribution on both sides, with equal amplitude and opposite signs, respectively, to ensure the continuity of the electric field through the aperture. The integral equation which imposes the continuity of the magnetic field follows from the relation $\mathbf{H}_{\mathrm{S}}^{+}(\mathbf{M})+\mathbf{H}_{\text {imp }}=\mathbf{H}_{\mathrm{S}}(-\mathbf{M})$, where the superscript + and - refer to the Green's function of the upper and lower semi-infinite region, respectively. Following the same procedure such as the patch-type FSS, the GSM entries, for dielectric stratification in Fig.2, are given by [6], [7]

$$
\begin{aligned}
& \overline{\bar{S}}_{11}=\overline{\bar{\Gamma}}_{1, s c}-\overline{\bar{Z}}_{F W} \overline{\bar{Y}}_{t 1} ; \\
& \overline{\bar{S}}_{21}=\overline{\bar{Z}}_{F W} \overline{\bar{Y}}_{t 1} ; \\
& \overline{\bar{S}}_{12}=\overline{\bar{Z}}_{F W} \overline{\bar{Y}}_{t 2} ; \\
& \overline{\bar{S}}_{22}=\overline{\bar{\Gamma}}_{2, s c}-\overline{\bar{Z}}_{F W} \overline{\bar{Y}}_{t 2} ;
\end{aligned}
$$

in which $\overline{\bar{Z}}_{F W}=\overline{\bar{P}}_{A}\left(\bar{P}^{H} \overline{\bar{Y}}_{G F} \overline{\bar{P}}^{-1} \overline{\bar{P}}_{A}^{H}\right.$, and the $\overline{\bar{P}}$ entries are the $\mathrm{TM} / \mathrm{TE}$ components of the basis functions spectra, sampled at the FW wavenumbers. The matrix $\overline{\bar{P}}_{A}$ is the relevant matrix educed to the accessible modes, and the other matrices are the same as those for the patch-type FSS case. Furthermore $\overline{\bar{\Gamma}}_{1, s c}$ and $\overline{\bar{\Gamma}}_{2, s c}$ are scattering matrices at the interface 1 and 2 (Fig. 2) in absence of FSS aperture (i.e. short circuiting the apertures), $\overline{\bar{Y}}_{t 1}$ and $\overline{\bar{Y}}_{t 2}$ are the trans-admittance matrices linking the currents in one side of the FSS plane to the voltages of the opposite side of the plane. A similar coherent representation is derived for the junction associated to the periodic array of open-ended waveguides, with input ports associated to waveguide modes and output ports associated with Floquet modes. The GSM entries are given by

$$
\begin{aligned}
& \overline{\bar{S}}_{11}=-\overline{\bar{I}}+2 \overline{\bar{P}}_{W G, A} \overline{\bar{Y}}_{M o M, a}^{-1} \overline{\bar{P}}_{W G, A}^{H} \overline{\bar{Y}}_{W G} ; \\
& \overline{\bar{S}}_{21}=2 \overline{\bar{P}}_{F W, A} \overline{\bar{Y}}_{M O M, a}^{-1} \overline{\bar{P}}_{W G, A}^{H} \overline{\bar{Y}}_{W G} ; \\
& \overline{\bar{S}}_{12}=2 \overline{\bar{P}}_{W G, A} \overline{\bar{Y}}_{M o M, a}^{-1} \overline{\bar{P}}_{F W, A}^{H} \overline{\bar{Y}}_{H S} ; \\
& \overline{\bar{S}}_{22}=-\overline{\bar{I}}+2 \overline{\bar{P}}_{F W, A} \overline{\bar{Y}}_{M o M, a}^{-1} \overline{\bar{P}}_{F W, A}^{H} \overline{\bar{Y}}_{H S} ;
\end{aligned}
$$

where $\stackrel{\bar{Y}}{Y}_{M o M, a}^{-1}=\left(\bar{P}_{W G}^{H} \overline{\bar{Y}}_{G F, a} \overline{\bar{P}}_{W G}\right)^{-1}$ and all the matrices are 
defined in analogy with the aperture-type FSS, but with reference on both the internal waveguide modes (subscript WG) and external FW modes (subscript FW) .

\section{Interpolation of Scattering Matrix}

In order to design the FSS geometry to be integrated with the phased array antenna, it is necessary to predict the angular response of the structure in the frequency range of interest. This can prejudice the computational time saving, due to the large amount of required numerical simulations. To reduce computational time, a fast interpolation algorithm for the scattering matrix has been proposed. Due to the complex poles-zeros nature of the scattering matrix entries, we implemented an adaptive sampling algorithm for the generation of a bivariate interpolation, based on a Thiele-type branched continued fraction representation described in [8]. The interpolation can be performed at fixed frequency to synthesize the angular behavior (beam scanning) as well as for both angular and frequency dependence. In the single-frequency case, the interpolated function (the elements of the scattering matrix) is a bivariate function of the spherical coordinates $(\theta, \varphi)$, representing the direction of incidence. Future extensions may include the frequency interpolation in order to obtain fast and efficient models for wideband applications. The convergence of the iterative process is tested by using cumulative residual functions defined as

$$
\begin{aligned}
& E_{k}(\varphi, \theta, \omega)=\sum_{n=1}^{N} \sum_{m=n}^{N} E_{k, n m}(\varphi, \theta, \omega)= \\
& =\sum_{n=1}^{N} \sum_{m=n}^{N} \frac{\left|R_{k, n m}(\varphi, \theta, \omega)-R_{k-1, n m}(\varphi, \theta, \omega)\right|^{2}}{\left(1+\left|R_{k, n m}(\varphi, \theta, \omega)\right|\right)^{2}}
\end{aligned}
$$

where $R_{k, n m}(\varphi, \theta, \omega)$ is the $k^{\text {th }}$ order approximation of the $n m^{\text {th }}$ scattering element.

\section{Validation and Numerical Results}

Before facing the design process, a series of numerical tests have been performed to validate the developed numerical architecture and the relevant software tool. The validation is obtained by comparison with the results by the commercial software CST ${ }^{\mathrm{TM}}$ Microwave Studio ${ }^{\mathrm{TM}}$.

The first configuration is represented in Fig. 4. The structure consists of a phased array of open-ended waveguides (aperture width $a=32 \mathrm{~mm}$, height $b=12 \mathrm{~mm}$ ), arranged on a rectangular grid with periodicity $d_{x}=35 \mathrm{~mm}$ and $d_{y}=15 \mathrm{~mm}$ along the $x$ and the $y$ axis, respectively. A patch-type cross-dipole FSS is placed at a distance $l_{1}=19 \mathrm{~mm}$ over the array plane. Two cases have been considered: an 'ideal' free-standing FSS, and an FSS printed on the back of a $3 \mathrm{~mm}$ thick dielectric layer with relative permittivity $\varepsilon_{r}=3$. The analysis has been performed in the frequency range from 4.8 to $8 \mathrm{GHz}$, for a broadside incident plane wave.

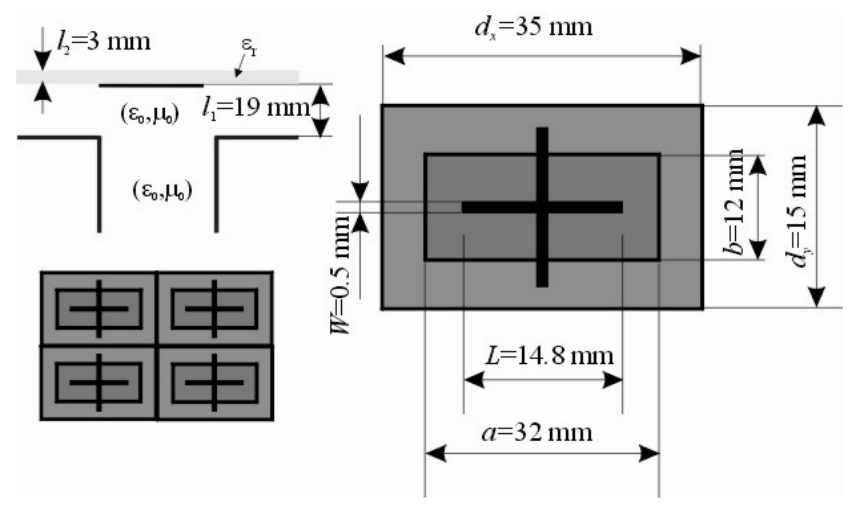

Fig. 4. Phased array of open-ended waveguides integrated with a cross-dipoles FSS: geometrical parameters.

The simulation parameters of the software tool are: 17 basis function for the expansion of the magnetic current on the waveguide aperture, 25 basis function for the expansion of the electric current on the cross-dipole, and maximum index of the FW modes (used to fill the MoM matrix) equal to 23 .

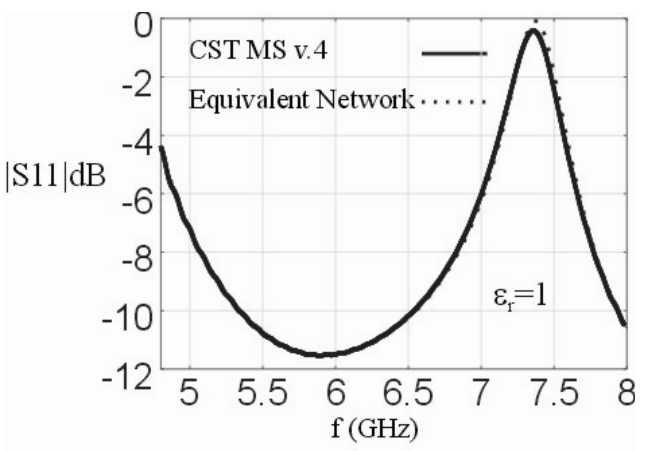

(a)

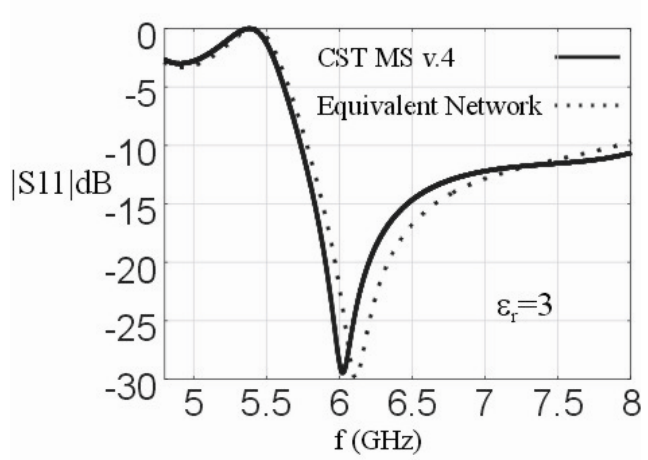

(b)

Fig. 5. Reflection coefficient (broadside beam) for the structure in Fig. 4: comparison between the results from the tool based on the network model (dotted line) and those from CST MS 4.0 (solid line): (a) free-standing FSS; (b) 3mm thick dielectric-backed FSS.

The results of the simulation have been compared with those from CST MS, and are shown in Fig. 5, which presents the magnitude of the active reflection coefficient 
at the waveguide port $\mathrm{TE}_{10}$ of the mode. Excellent agreement has been obtained, with a slight frequency shift when the dielectric layer is present.

The second configuration is presented in Fig. 6 and Fig. 8. This structure is formed by a cascade of two identical loop-type FSS, inside an appropriate dielectric stratification, placed over a phased array of open-ended waveguides, arranged on a skew grid. This configuration is designed to obtain a rejection of the third harmonic to respect the operative bandwidth of the array. In particular the phased array of open-ended waveguides is sized to have an operative bandwidth between 5 to $6 \mathrm{GHz}$ (Fig. 8) and therefore the rejection frequencies range is between 15 to $18 \mathrm{GHz}$. To obtain this so large stop band a pair of FSSs separated by suitable dielectric layer has been used. To design a stop band screen for a broad frequency range through multiple FSSs we use the same strategy as in [9]. The two dielectric layers with $\varepsilon_{\mathrm{r}}=10.2$ and $\varepsilon_{\mathrm{r}}=2.94$ realize a two-section binomial transformer to match to free-space on the operative impedance of the open-ended waveguide array over a large operative bandwidth. To represent the electric current of the loop-type FSS for the spectral domain MoM, we chose spectral basis function like those associated to the coaxial waveguide modes [10].
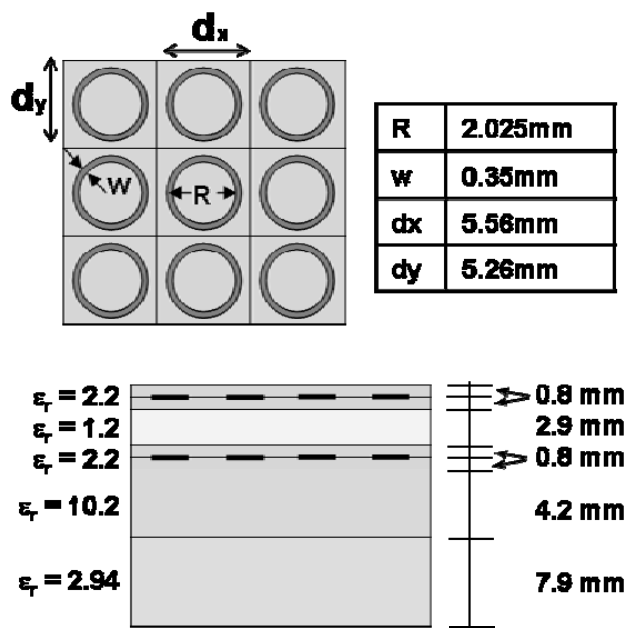

Fig. 6. Geometrical dimensions of the loop-type FSS and relative dielectric stratifications.

Fig. 7 presents the amplitude in $\mathrm{dB}$ of the reflection coefficient for the $\mathrm{TE}_{\mathrm{z}}$ polarization (with $\mathrm{z}$ normal to the stratification) of the isolated band-stop screen for various angles of incidence. For broad scan, the structure exhibits a passband in the operative band of the array and a stop-band around the third harmonic of the array operating frequency.

In a second set of results, the stop-band arrangement in Fig. 6 is integrated with the open-ended waveguide array, whose rectangular waveguide elements are showed in Fig. 8, in a second plane to respect the circular loop FSS. Fig. 9 presents the magnitude of the $\mathrm{TE}_{10}$ reflection coefficient as a function of frequency for different mainbeam scan-angles on the $\mathrm{H}$-plane. The input active impedance of the array satisfactorily remains well matched at $-12 \mathrm{~dB}$ in a band of $1 \mathrm{GHz}$ around $5.5 \mathrm{GHz}$ for a scan range of 30 degrees (the results in the stop band at the third harmonic, not presented here, are also successful as expected).

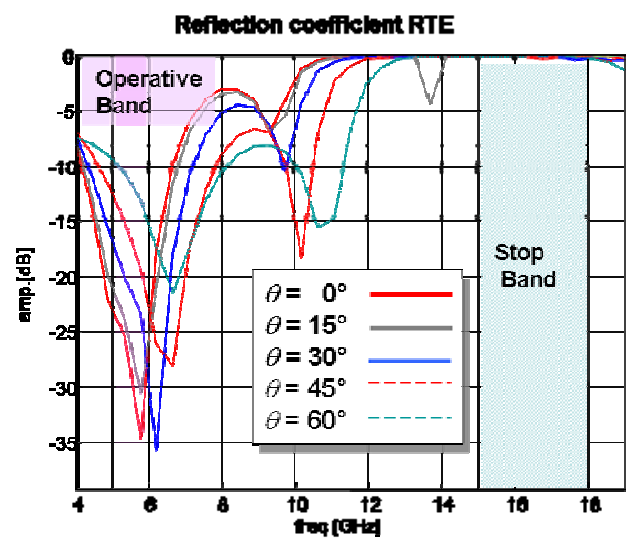

Fig. 7. Magnitude of the reflection coefficient for the $\mathrm{TE}_{\mathrm{z}}$ polarization (with $\mathrm{z}$ normal to the stratification) for the arrangement in Fig. 6, for various angles of incidence.

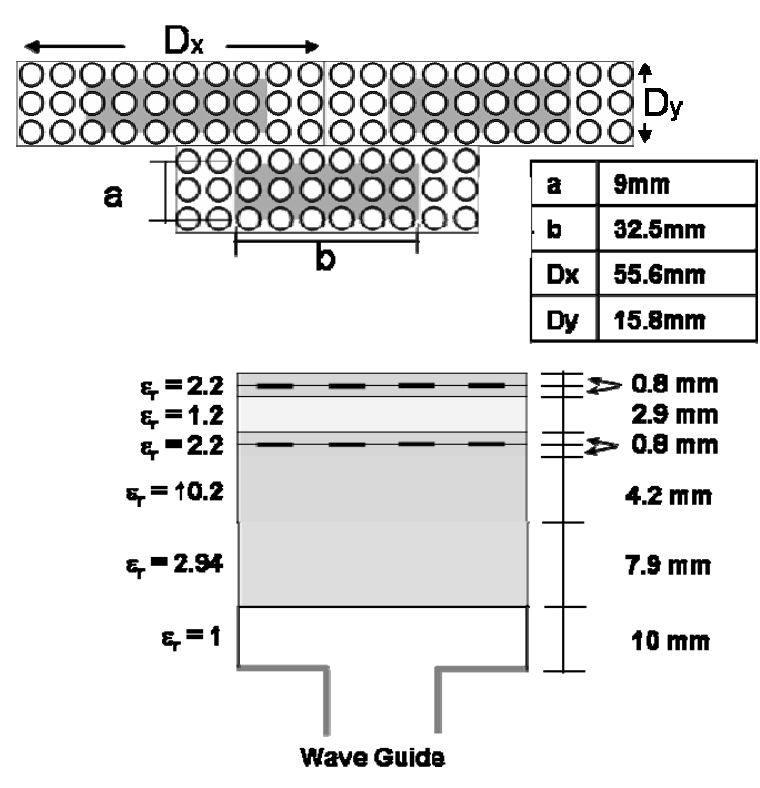

Fig. 8. Top end lateral view of phased array of open-ended waveguides integrated with a stop-band loop-type FSS: geometrical parameters and dielectric stack-up.

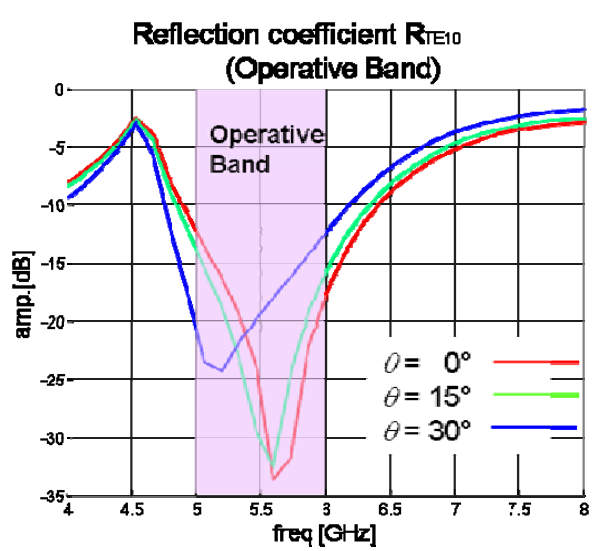

Fig. 9. Magnitude of the $\mathrm{TE}_{10}$ reflection coefficient for the structure in Fig. 8 as a function of frequency for different main-beam scan-angles on the H-plane. 


\section{Conclusions}

In this paper we have presented an efficient and accurate model to design multilayer frequency selective surfaces (FSSs) integrated with a phased array of open-ended waveguides. The main scope of the presence of the FSS is to reject the third harmonic, to avoid disturbance on the apparatus around. The method uses the representation of an equivalent multi-mode transmission line network, where each interface is characterized by an equivalent Generalized Scattering Matrix (GSM), computed through spectral domain full-wave MoM analysis. The scheme is first applied to various numerical architectures and then successfully compared with the results achieved with commercial softwares.

\section{References}

[1] MITTRA, R., CHAN, C. H., CWIK, T. Techniques for analyzing frequency selective surfaces - A review. Proceedings of the IEEE, 1988, vol. 76, no. 12 .

[2] MONNI, S., GERINI, G. A novel technique for the design of frequency selective structures integrated with a waveguide array. In IEEE APS International Symposium, 2004, vol. 2, p. 2179-2182.

[3] KNITTEL, G. H., HESSEL, A., OLINER, A. A. An element pattern nulls in phased arrays and their relation to guided waves. Proceedings of the IEEE, 1968, vol. 56, no 11.

[4] MOSCA, S., MERCANTI, B., FANI, A. A graphical method to design radiating elements for phased array antennas. In IEEE $12^{\text {th }}$ International Conference on Antennas and Propagation, 2003.

[5] ROZZI, T. E., MECKLENBRAUKER, W. F. G. Wide-band network modeling of interacting inductive irises and steps. IEEE Transactions on Microwave Theory and Techniques, 1975, vol. 23, no. 2.

[6] MACI, S., CUCINI, A. FSS-based complex surfaces. In Electromagnetic Metamaterials: Physics and Engineering Aspects. Edited by N. Engheta and R. W. Ziolkowski, Wiley InterScience, 2006.

[7] TASCONE, R., ORTA, R. Frequency Selective Surfaces - Analysis and Design. Ch. 7, edited by J. C. Vardaxoglou, Research Studies Press Ltd., 1997.

[8] LEHMENSIEK, R., MEYER, P. Creating accurate multivariate rational interpolation models of microwave circuits by using efficient adaptive sampling to minimize the number of computational electromagnetic analyses. IEEE Transactions on Microwave Theory and Techniques, 2001, vol. 49, no. 8, p. 1419-1430.

[9] MUNK, B. A. Frequency Selective Surfaces. Ch. 8. John Wiley \& Sons, Inc, 2000.

[10] MARCUVITZ, N. Waveguide Handbook. Boston Technical Publishers, Inc., p. 72-80, 1964.

\section{About Authors ...}

Francesco CAMINITA (*1976 in Montevarchi, Arezzo, Italy) received the Laurea degree (cum laude) in telecommunications engineering from the University of Siena, Italy, in 2005, where he is working toward the Ph.D. degree in electromagnetic engineering. Since 2006, he has been involved in projects funded by the European Space Agency (ESA) and the European Community regarding software for antenna simulation. His research interests are in the area of periodic structures, frequency selective surfaces, electromagnetic band-gap structures, artificial surfaces and holographic antennas.

Alessio CUCINI (S’00-M’03, *1973 in Poggibonsi, Italy) received the Laurea degree (cum laude) in telecommunications engineering and the Ph.D. degree in electromagnetic engineering from the University of Siena, Italy, in 1999 and 2003, respectively. He is currently a Research Associate in the Dept. of Information Engineering, University of Siena. He is also with WaveComm S.r.l., a company operating in the fields of electromagnetic software, antennas, sensors, and RF and microwave systems. Since 1999, he has been involved in projects funded by ESA and the European Community regarding software for antenna simulation. His research interests are in the area of applied electromagnetics, focused on numerical and asymptotic methods for array antennas, frequency selective surfaces, EBG structures, artificial surfaces and microstrip antennas.

Stefano MACI (*1961 in Rome) received his laurea degree (cum laude) in Electronic Engg. from the University of Florence, Italy, in ' 87. Since '98 he is with the University of Siena, Italy, where he presently is a Full Professor. His research interests are focused on Electromagnetism, with emphasis to high-frequency and numerical methods for antennas. He was a co-author of an Incremental Theory of Diffraction for the description of a wide class of electromagnetic scattering phenomena at high frequency, and of a diffraction theory for the high frequency analysis of large truncated periodic structures. His research interests involve high-frequency methods for radiation and scattering problems, hybrid integral equation/asymptotic methods, large phased array antennas, planar antennas and multilayer structures, reflector antennas and feed horns, electromagnetic bandgap structures and artificial surfaces. $\mathrm{He}$ is the project leader of the "European school of antennas" (ESoA), a collection of courses devoted to PhD students framed in a European Marie Curie Action program. He was associate Editor of IEEE Trans. EMC ('99-'01). He was presently a member of the Technical Advisory Board of the URSI Commission B, and a member of the advisory board of the Italian PhD school of Electromagnetism. In 2003 he was elected Fellow of IEEE for his scientific contributions on diffraction theory of periodic printed structures in stratified media. He was a guest editor of the special issue "Artificial magnetic conductor, soft/hard surfaces and other complex surfaces" of IEEE Trans. Antennas Propag. (Jan. '05), a guest Editor of the Special Issue "Electromagnetic wave propagation in complex environments: a tribute to Professor L.B. Felsen” of IEEE Trans. Antennas Propag. (June '07). He is presently an associate Editor of IEEE Trans. Antennas Propag. In '06 was awarded by the Applied Computational Electromagnetic Society the best journal paper award. He is principal author or co-author of about 100 papers published in international journals and book Chapters, among which about 60 papers on IEEE journals, and about 300 papers in proceedings of international conferences. 